\title{
N.M.R. investigations on the alkali naphthalene ion pairs
}

\author{
by B. M. P. HENDRIKS, G. W. CANTERS†, C. CORVAJAt, \\ J. W. M. DE BOER and E. DE BOER \\ Department of Physical Chemistry, University of Nijmegen, \\ Nijmegen, The Netherlands
}

(Received 6 April 1970)

\begin{abstract}
Nuclear magnetic resonance has been carried out on all nuclei with nonzero magnetic moments present in the alkali ion pairs of deuterated and nondeuterated naphthalene radical anion in 1,2-dimethoxyethane. Both the sign and magnitude of the hyperfine splitting constant (h.f.s.c.) were measured, the alkali h.f.s.c. being studied as a function of the temperature. The $\mathrm{Li}$ and $\mathrm{Na}$ h.f.s.c. appear to be positive, those of $\mathrm{Rb}$ and $\mathrm{Cs}$ are negative, whereas that of $\mathrm{K}$ changes sign with temperature.

The proton and deuterium linewidths were studied as a function of the concentration and were found to vary linearly with the reciprocal of the concentration, pointing to the predominance of the Fermi contact interaction.

An analysis of the alkali linewidth is given in terms of the intramolecular relaxation processes. Especially in cases where two isotopes could be studied, i.e. $\left({ }^{1} \mathrm{H},{ }^{2} \mathrm{D}\right),\left({ }^{6} \mathrm{Li},{ }^{7} \mathrm{Li}\right)$ and $\left({ }^{85} \mathrm{Rb},{ }^{87} \mathrm{Rb}\right)$, a quantitative interpretation is presented.
\end{abstract}

\section{INTRODUCTION}

Since the pioneering work of Atherton and Weissman on the association between sodium and naphthalenide ions [1], many papers have appeared on E.S.R. studies of ion pairs and much information on alkali metal hyperfine splitting constants (h.f.s.c.) has been collected [2]. The alkali metal hyperfine coupling was found to be very sensitive to the solvent and the temperature, suggesting that transfer of spin density from the aromatic radical to the metal proceeds in a complex manner. Unfortunately E.S.R. experiments only give the absolute value of the h.f.s.c., whereas in determining the mechanism of spin transfer the sign of the h.f.s.c. is also of importance.

De Boer [3], in an E.S.R. study of the pyracene anion radical, observed that the $\mathrm{Cs}$ and $\mathbf{R b}$ h.f.s.c. exhibited an anomalous temperature dependence. He suggested that the h.f.s.c. of these ions could be negative as well as positive.

Negative alkali spin densities were also proposed by Dodson and Reddoch [4] and Hirota [5], in studying the alkali naphthalene ion pairs. They noticed that regularly decreasing plots of spin density at the alkali nucleus (calculated as the ratio of the observed hyperfine splitting in the ion pair to that of the atom in the gas phase [6]) versus the radius $r$ of the alkali ion, were obtained if the spin density at the $\mathrm{Rb}$ and Cs nucleus was taken negative. This correlation between spin density and ionic radius has also been observed for other systems, e.g. alkali anthracenides [5],

† Present address: Department of Chemistry, University of North Carolina, Chapel Hill, N.C. 27514, U.S.A.

† Nato post-doctoral fellow 1968-1969. Present address: Department of Physical Chemistry, University of Padua, Italy. 
and the ion pairs of 1,2-naphthosemiquinone and acenaphthenesemiquinone [7]. To obtain smooth plots, the sign of the spin density at the alkali nuclei had to be taken negative for $\mathrm{Cs}-$ anthracenide and for $\mathrm{Rb}$ and $\mathrm{Cs}$-acenaphthenesemiquinone.

In order to test the hypotheses about the sign and the sign reversal of the alkali h.f.s.c., Canters et al. [8] investigated some alkali radical ion pairs by N.M.R.

This method has some advantages above the E.S.R. technique. As each group of equivalent nuclei in a radical ion pair is characterized by only one single N.M.R. signal, the N.M.R. spectrum of a radical ion pair will be more easily interpretable than the corresponding E.S.R. spectrum of the ion pair. A special advantage is that both sign and magnitude of the h.f.s.c. can be inferred directly from the sign and magnitude of the Fermi contact shift measured in the N.M.R. spectrum of the nucleus. The N.M.R. data show that for the alkali biphenyl ion pairs in 1,2dimethoxyethane (DME) the spin density at the $\mathrm{Li}$ and $\mathrm{Na}$ nuclei is positive, whereas a negative spin density is obtained at the $\mathrm{K}$ and $\mathrm{Rb}$ nuclei. For Cs ion pairs measured in polyglycoldimethylethers, always a negative sign was found. Moreover, information was obtained on the structure of the ion pairs from an analysis of the alkali linewidth [9].

In this paper we present the results of a N.M.R. study on the alkali naphthalene ion pairs. Both proton and alkali resonance on all commonly occurring isotopes were carried out. Throughout the investigations DME was used as solvent. The $\mathrm{Na}$-naphthalene $(\mathrm{Na}-\mathrm{Nl})$ complex was also studied in tetrahydrofuran (THF). Besides proton resonance, deuterium resonance measurements were carried out on completely deuterated samples.

In summary, the following results were obtained:

With regard to the proton resonance, the signals of both the $\alpha$ and $\beta$ protons were observed. The h.f.s.c. derived from the contact shifts in the ${ }^{1} \mathrm{H}$ spectrum were in good agreement with the E.S.R. h.f.s.c. From the linewidth, values for the correlation times were obtained. The linewidth proved to be proportional to the inverse of the concentration of negative ions, indicating the predominance of the Fermi-contact interactions in determining the linewidths.

The $2 \mathrm{D}$ resonance spectra were nicely resolved [10]. In mixed samples containing normal and deuterated $\mathrm{Nl}$, we were able to determine the quadrupole contribution to the linewidth: its contribution to the $\alpha$ and $\beta$ 2D peaks was almost equal.

The alkali resonances revealed a positive spin density at the $\mathrm{Li}$ and $\mathrm{Na}$ nucleus and a negative value at the $\mathrm{Rb}$ and $\mathrm{Cs}$ nucleus, whereas a sign reversal was observed for $\mathrm{K}$. Until now a satisfactory theory explaining the various observations has been lacking. However, in a subsequent article we will give a detailed explanation for these phenomena. The measured alkali linewidths, especially those of ${ }^{6} \mathrm{Li},{ }^{7} \mathrm{Li}$ ${ }^{85} \mathrm{Rb}$ and ${ }^{87} \mathrm{Rb}$, enabled us to determine the contributions of the various relaxation mechanisms to the linewidth. Moreover information was obtained on the nature of the ion pairs in solution.

\section{EXPERIMENTAL}

All measurements were performed on a Varian DP 60 external lock (EL) spectrometer, equipped with a V-4311 $60 \mathrm{MHz}$ unit for proton N.M.R. and a variable frequency transmitter $\mathrm{V}-4210$ for the alkali and deuterium resonances. The frequency of the V-4210 unit was stabilized by crystal stabilizers at $15 \cdot 1 \mathrm{MHz}$ 
for the ${ }^{7} \mathrm{Li},{ }^{23} \mathrm{Na}$ and ${ }^{87} \mathrm{Rb}$ resonances, at $9 \cdot 1 \mathrm{MHz}$ for ${ }^{2} \mathrm{D}$ and ${ }^{6} \mathrm{Li}$, at $8 \cdot 0 \mathrm{MHz}$ for ${ }^{133} \mathrm{Cs}$, at $5.9 \mathrm{MHz}$ for ${ }^{85} \mathrm{Rb}$ and at $2.9 \mathrm{MHz}$ for ${ }^{39} \mathrm{~K}$ resonance. The frequency was counted with a Hewlett-Packard $5245 \mathrm{~L}$ frequency counter and the magnetic field was measured with an AEG' gaussmeter. The temperature of the sample, controlled by a V-4540 unit, was measured with a thermocouple.

The samples were prepared by standard techniques [11] using a vacuum line. The extent of the reduction was determined by measuring the shift of the solvent peaks with respect to their position in an unreduced N1 solution. Reduction was stopped when the maximum shift was reached. The spectrometer was operated in the EL mode when following the reduction by looking at the solvent shift; the wide line (WL) mode was used to record the proton, deuterium and alkali nuclei resonances and the high resolution (HR) mode was employed to record the narrow resonances of ${ }^{6} \mathrm{Li},{ }^{7} \mathrm{Li}$ and also ${ }^{2} \mathrm{D}$. Stable solutions in the concentration range from 0.3 to $2 \cdot 3 \mathrm{M}$ were obtained for all alkali metals in DME and for $\mathrm{Na}$ in THF. Alkali N.M.R. spectra are recorded every $10^{\circ} \mathrm{C}$ in the temperature range from $-60^{\circ}$ to $+100^{\circ} \mathrm{C}$. Proton and deuterium N.M.R. was performed only at $+30^{\circ} \mathrm{C}$.

The contact shifts were corrected for the bulk susceptibility and the concentration difference between sample and reference as described in ref. [12]. As

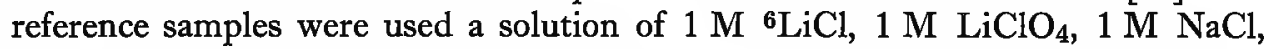
$1 \mathrm{M} \mathrm{KCl}, 1.5 \mathrm{M} \mathrm{RbCl}$ and $2.4 \mathrm{M} \mathrm{CsCl}$ in $\mathrm{H}_{2} \mathrm{O}$. The linewidths were corrected for modulation broadening $[13,14]$ and for broadening by intermolecular interactions. The latter correction, determined by the paramagnetic particles in the solution, was obtained by measuring the broadening of the solvent proton signal.

Measurements as a function of radical concentration were carried out by concentrating the sample. The volume of the solutions was determined with an accuracy between 2 and 5 per cent by means of a calibrated tube.

\section{GenERAL CONSIDERATions}

\subsection{Contact and paramagnetic shifts}

N.M.R. spectra of paramagnetic particles are modified in two ways with respect to the spectra of the corresponding diamagnetic species. Firstly the resonance lines are broadened and secondly the lines are shifted by the Fermi contact interaction.

The Fermi contact shift $[8,9], \delta_{\mathbf{c}^{0}}$ expressed in gauss, is given by

$$
\delta_{\mathrm{c}}{ }^{0}=-\frac{\gamma_{\mathrm{e}}}{\gamma_{\mathrm{N}}} a \frac{\gamma_{\mathrm{e}} \hbar H_{\mathrm{p}}}{4 k T}
$$

where $a$ denotes the h.f.s.c. in gauss and $H_{\mathrm{p}}$ the field at which resonance occurs; the other symbols have their usual meaning.

Because

$$
a=\frac{8 \pi}{3} \gamma_{\mathrm{N}} \hbar \rho\left(\mathbf{r}_{\mathrm{N}}\right)
$$

where $\rho\left(r_{N}\right)$ is equal to the spin density at the nucleus $N$ with radius vector $r_{N}$, the contact shift $\delta_{\mathrm{c}}{ }^{0}$ is independent of $\gamma_{\mathrm{N}}$. From the direction of the shift the sign of the spin density can be inferred; a high field shift indicates a negative $\rho\left(\mathbf{r}_{N}\right)$, a low field shift indicates a positive $\rho\left(r_{N}\right)$.

In a partly reduced solution of negatively charged paramagnetic ions, the observed contact shift $\delta_{\mathrm{c}}$ is given by

$$
\delta_{\mathrm{c}}=f_{\mathrm{p}} \cdot \delta_{\mathrm{c}}{ }^{0}
$$


where $f_{\mathrm{p}}$ is the fraction of negative particles. By measuring $\delta_{\mathrm{c}}$ as a function of the degree of the reduction, $f_{\mathrm{p}}$, one should be able to construct linear plots of $\delta_{\mathrm{c}}$ against $f_{\mathrm{p}}$. From the slope of these straight lines the h.f.s.c. $a$ can be determined. A difficulty in this procedure is the determination of $f_{\mathrm{p}}$. In principle $f_{\mathrm{p}}$ could be determined from the paramagnetic shift of the solvent resonance signal $\left(\delta_{\mathrm{s}}\right)$, caused by the paramagnetic particles. If no special effects occur [16], $\delta_{\mathrm{s}}$ should be linearly related to the susceptibility change of the solution when paramagnetic ions are introduced. For the solutions under investigation, we noticed that the measured $\delta_{\mathrm{s}}$ was always smaller than the theoretical value, calculated with the Langevin susceptibility expression [17]. To avoid this difficulty we have taken as a measure for the reduction degree [12]

$$
f_{\mathrm{p}}=\frac{\delta_{\mathrm{g}}}{\delta_{\mathrm{s}, \max }}
$$

where $\delta_{\mathrm{s}, \max }$ is the maximum solvent shift at the end of the reduction. Experiments carried out in this laboratory, by Smid and Grotens [18], have shown that $\delta_{\mathrm{s}, \max }$ is linearly related to the concentration of paramagnetic particles. Moreover, they found that if small amounts of a polar solvent (glymes) are added to solutions in THF, the THF resonance signals shifted to their theoretical expected positions, whereas the peaks of the polar solvent in some instances were shifted by more than $200 \mathrm{~Hz}$ to low field.

These experiments prove that deviations from the Langevin formula are caused by contact interactions between the paramagnetic particles and the solvent molecules. Finally the h.f.s.c., derived with the aid of equation (4), were in good agreement with the h.f.s.c., obtained by E.S.R. This provides indirect evidence for the correctness of equation (4).

\subsection{Broadening mechanisms}

Generally the linewidth of a resonance peak will depend on inter and intramolecular interactions. For a resonating nucleus in a paramagnetic molecule the intramolecular interactions are by far the most important. In our case the linewidth parameter $T_{2}$ will be governed by a sum of three intramolecular contributions,

$$
T_{2}^{-1}=\left(T_{2}^{-1}\right)_{\text {F.C. }}+\left(T_{2}^{-1}\right)_{\mathrm{D}}+\left(T_{2}^{-1}\right)_{\mathrm{Q}},
$$

where the three terms on the right-hand side represent the contributions due to the Fermi contact interaction, the anisotropic electron-nucleus dipolar interaction and the quadrupolar interaction, respectively. For a radical in solution, subjected to rapid Brownian motion, the following expressions for $T_{2}{ }^{-1}$ have been derived for the three different types of interactions [19-21]:

$$
\begin{aligned}
\left(T_{2}^{-1}\right)_{\text {F.C. }} & =\frac{1}{3} a^{2} \gamma_{\mathrm{e}^{2}} S(S+1)\left\{\tau_{\mathrm{e}}+\frac{\tau_{\mathrm{e}}}{1+\omega_{\mathrm{e}}{ }^{2} \tau_{\mathrm{e}}{ }^{2}}\right\} \\
\left(T_{2}^{\prime-1}\right)_{\mathrm{D}} & =\frac{1}{15} \frac{\gamma_{\mathrm{e}}^{2} \gamma_{\mathrm{N}} \hbar^{2}}{r^{6}} S(S+1)\left\{7 \tau_{\mathrm{d}}+\frac{13 \tau_{\mathrm{d}}}{1+\omega_{\mathrm{e}}^{2} \tau_{\mathrm{d}}{ }^{2}}\right\}, \\
\left(T_{2}^{-1}\right)_{\mathrm{Q}} & =\frac{3}{40} f(\mathrm{I}) \cdot\left(\frac{e^{2} Q q}{\hbar}\right)^{2}\left(1+\frac{\eta^{2}}{3}\right) \tau_{\mathrm{r}},
\end{aligned}
$$


where $\tau_{\mathrm{e}}$ is the electron correlation time, $\tau_{\mathrm{d}}$ the dipolar correlation time defined by $\tau_{\mathrm{d}}{ }^{-1}=\tau_{\mathrm{e}}{ }^{-1}+\tau_{\mathrm{r}}{ }^{-1}, \tau_{\mathrm{r}}$ the rotational correlation time, $f(\mathrm{I})=(2 I+3) / I^{2}(2 I-1), e Q$ the quadrupole moment, $e q$ the electric field gradient and the other symbols have their usual meaning. Equation (7) has been derived for the interaction between magnetic point dipoles, separated by a distance $r$.

Under the conditions of our experiment the correlation times are of the order of $10^{-10}$ to $2 \times 10^{-11} \mathrm{~s}[12,15]$, whereas the electron resonance frequency $\omega_{\mathrm{e}}$ is about $3 \times 10^{11} \mathrm{rads} / \mathrm{s}$. It follows then, that only secular parts of the various relaxation mechanisms contribute to $T_{2}^{-1}$, so that equations (6)-(8) can be simplified to $\left(S=\frac{1}{2}\right)$ :

$$
\begin{aligned}
\left(T_{2}{ }^{-1}\right)_{\mathrm{F} . \mathrm{C} .} & =\frac{1}{4} \gamma_{\mathrm{e}}{ }^{2} a^{2} \tau_{\mathrm{e}}, \\
\left(T_{2}{ }^{-1}\right)_{\mathrm{D}} & =\frac{7}{20} \frac{\gamma_{\mathrm{e}^{2} \gamma_{\mathrm{N}} \hbar^{2}}}{r^{6}} \tau_{\mathrm{d}} \\
\left(T_{2}{ }^{-1}\right)_{\mathrm{Q}} & =\frac{3}{40} f(I)\left(\frac{e^{2} Q q}{\hbar}\right)^{2} \tau_{\mathrm{r} .} \quad(\eta=0)
\end{aligned}
$$

The linewidths should now be proportional to a particular correlation time. These correlation times depend on the radical concentration $c$, the absolute temperature $T$ and the viscosity $\eta$. According to the Debye-Einstein model [22] the dependence of $\tau_{\mathbf{r}}$ on $\eta$ and $T$ is given by

$$
\tau_{\mathbf{r}} \sim \eta / T,
$$

while according to the model of Pake and Tuttle [23] the dependence of $\tau_{\mathrm{e}}$ on $c, T$ and $\eta$ is given by

$$
\tau_{\mathrm{e}} \sim \frac{\eta}{T} \cdot \frac{1}{c}
$$

From these relations it follows that the total linewidth will be proportional to $\eta / T$, if equations (9)-(11) are valid.

Briefly, a few comments should be made about the dependence of the viscosity on the concentration. This can be represented by [24]

$$
\eta(c, T)=\eta_{0}(T)\left(1+\lambda_{1} \sqrt{ } c+\lambda_{2} c+\lambda_{3} c^{2}\right)
$$

in which $\eta_{0}$ is the viscosity of the pure solvent and $\lambda_{1}, \lambda_{2}$ and $\lambda_{3}$ are numerical quantities. From the work of other investigators [25, 26] it follows that $\lambda_{1}, \lambda_{3} \ll \lambda_{2}$.

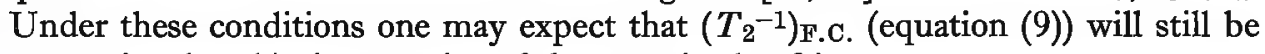
proportional to $1 / c$, irrespective of the magnitude of $\lambda_{2}$.

\section{Results}

\subsection{Proton magnetic resonance}

The ${ }^{1} \mathrm{H}$ N.M.R. experiments have been carried out on the system NaNl-h8 in $\mathrm{DME}$ as a function of the degree of reduction at $+30^{\circ} \mathrm{C}$. The reduction degree was determined by measuring the solvent shift, $\delta_{\mathrm{s}}$, and using equation (4) (naphthalene reduces quantitatively to $\mathrm{Nl}^{-}$in $\mathrm{DME}$ ).

In figure 1 we have plotted the Fermi contact shift, $\delta_{\mathfrak{c}}$, of the $\alpha$ and $\beta$ protons of naphthalene as a function of $f_{\mathrm{p}}$. Only at maximum reduction and high concentration of negative ions is it possible to get reliable data for the $\alpha$-proton 


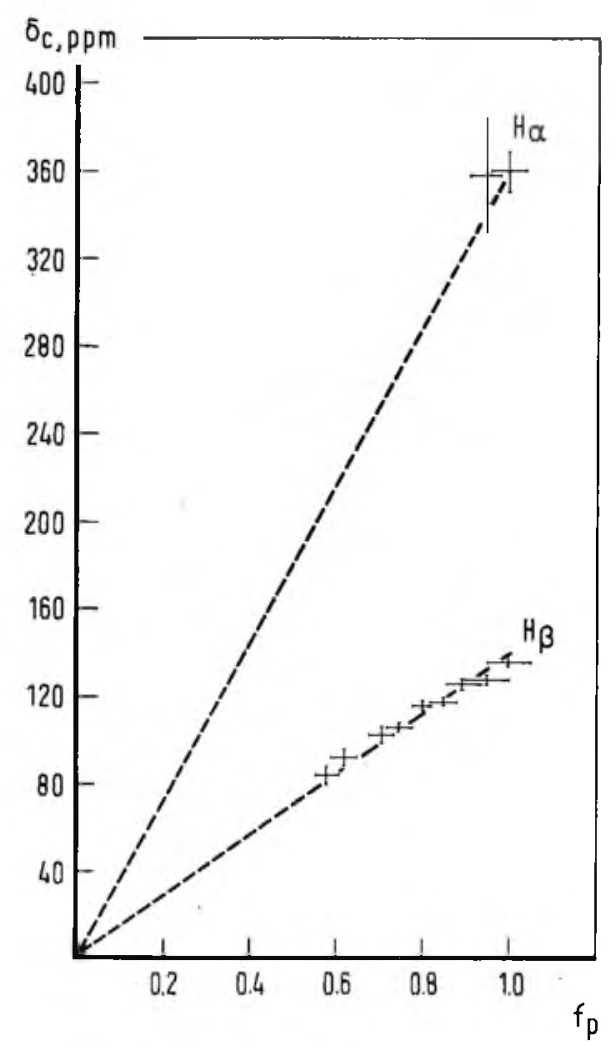

Figure 1. ${ }^{1} \mathrm{H}$ contact shift $\left(\delta_{\mathrm{c}}\right)$ versus degree of reduction $\left(f_{p}\right)$ for a $1.4 \mathrm{M}$ solution of naphthalene-h8 (N1-h8) in DME at $+30^{\circ} \mathrm{C}$.

resonance. The $\alpha$-proton peak is extremely broad, but narrows as the reduction approaches completion. From the slope of the straight line the h.f.s.c. was determined with the aid of equation (1). The mean values of the h.f.s.c., obtained from a series of different samples, are collected in table 1.

By concentrating the samples the linewidths of the absorption signals were measured as a function of the concentration. In figure $3, T_{2}{ }^{-1}$ is plotted against $1 / c$, and reasonable straight lines were obtained.

\begin{tabular}{|c|c|c|c|}
\hline & & $\alpha$ proton & $\beta$ proton \\
\hline $\begin{array}{l}\text { E.S.R. } \\
{ }^{1} \text { H N.M.R. } \\
{ }^{2} \text { D N.M.R. }\end{array}$ & $\begin{array}{l}a_{\mathrm{H}} \\
a_{\mathrm{H}} \\
T_{2^{-1}}\left(\mathrm{~s}^{-1}\right) \\
a_{\mathrm{D}} \cdot\left(\frac{\gamma_{\mathrm{H}}}{\gamma_{\mathrm{D}}}\right) \\
T_{2^{-1}}\left(\mathrm{~s}^{-1}\right)\end{array}$ & $\begin{array}{l}4 \cdot 925 \pm 0 \cdot 003 \\
-4 \cdot 87 \pm 0 \cdot 03 \\
{[6 \cdot 93 \pm 0 \cdot 20]} \\
(3 \cdot 30 \pm 0 \cdot 06) \times 10^{4} \\
{[6 \cdot 85 \pm 0 \cdot 26]} \\
-4 \cdot 80 \pm 0 \cdot 02 \\
{[6 \cdot 81 \pm 0 \cdot 23]} \\
(8 \cdot 5 \pm 0 \cdot 2) \times 10^{2} \\
{[5 \cdot 3 \pm 0 \cdot 3]}\end{array}$ & $\begin{array}{c}1 \cdot 820 \pm 0 \cdot 002 \\
-1 \cdot 85 \pm 0 \cdot 01 \\
{[1 \cdot 0]} \\
(0 \cdot 482 \pm 0 \cdot 009) \times 10^{4} \\
{[1 \cdot 0]} \\
-1 \cdot 84 \pm 0 \cdot 01 \\
{[1 \cdot 0]} \\
(1 \cdot 60 \pm 0 \cdot 05) \times 10^{2} \\
{[1 \cdot 0]}\end{array}$ \\
\hline
\end{tabular}

Table 1. Analysis of ${ }^{1} \mathrm{H}$ and ${ }^{2} \mathrm{D}$ N.M.R. spectrum of naphthalene-h8 and -d8 negative ion. 


\subsection{Deuterium magnetic resonance}

The 2D N.M.R. experiments were performed on the system NaN1-d8 in DME, to which a small amount of THF-d8 was added as an internal reference. The measurements were carried out at $+30^{\circ} \mathrm{C}$, as a function of both concentration and degree of reduction. In figures 2 and 3 the results are plotted in the same way as

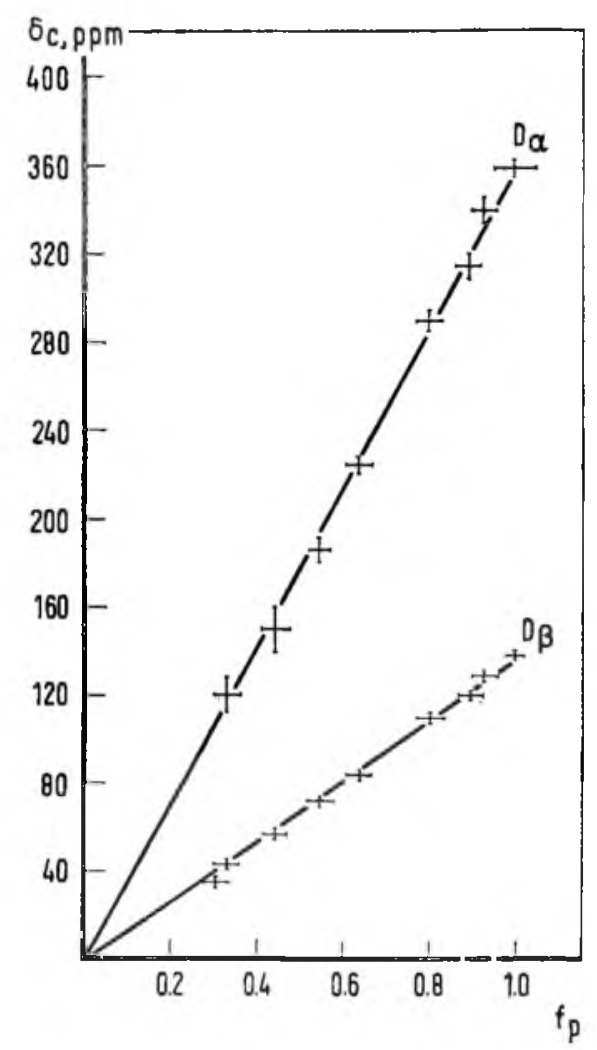

Figure 2, ${ }^{2} \mathrm{D}$ contact shift $\left(\delta_{\mathfrak{c}}\right)$ versus degree of reduction $\left(f_{\mathrm{p}}\right)$ for a $0.9 \mathrm{M}$ solution of Nl-d8 in DME at $+30^{\circ} \mathrm{C}$. About 5 volume per cent of $\mathrm{THF}-\mathrm{d} 8$ was added as internal reference.

was done for the ${ }^{1} \mathrm{H}$ data. The mean values of the h.f.s.c., again obtained from a number of samples, are listed in table 1.

Because of the small linewidth the $2 \mathrm{D}$ spectrum could also be recorded in the HR mode. The ratio between the full width at half height and the distance between points of extreme slope in the derivative was equal to $\sqrt{ } 3$ (within the accuracy of the measurements), pointing to a Lorentzian lineshape. The derivative curve was corrected for modulation amplitude [13] and modulation frequency [14] broadening.

\subsection{Mixed sample}

${ }^{1} \mathrm{H}$ and ${ }^{2} \mathrm{D}$ N.M.R. experiments were performed on a mixed sample, containing $1.63 \mathrm{M}$ of Nl-h8 and $0.62 \mathrm{M}$ of N1-d8. DME was used as solvent and $\mathrm{Na}$ as reducing agent. The measurements were carried out at $+30^{\circ} \mathrm{C}$, again using a small amount of THF-d8 as internal standard. 


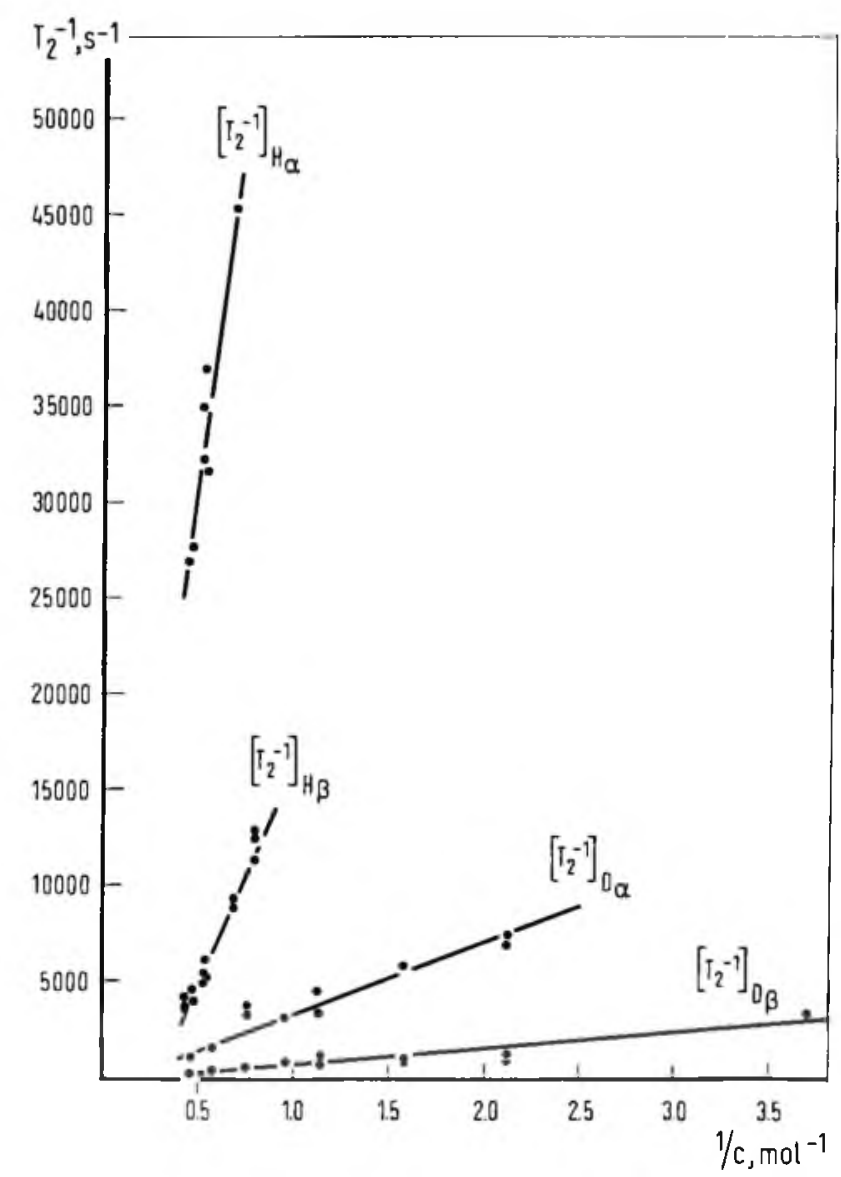

Figure 3. Linewidths of the $\alpha$ and $\beta$-proton peaks of NI-h8- and the $\alpha$ and $\beta$ deuterons of $\mathrm{Nl}-\mathrm{d} 8-$ versus the reciprocal of the concentration of $\mathrm{NI}^{-}$at $+30^{\circ} \mathrm{C}$.

Typical examples of observed spectra are depicted in figure 4. The linewidth data given in table 1 are derived from spectra obtained on this mixed sample.

\subsection{Alkali resonance}

All solutions were $1 \mathrm{M}$ in radical ions, except the $\mathrm{KNl}$ solution, where $1.8 \mathrm{M}$ was used to improve the signal to noise ratio. Throughout the investigations DME was used as solvent taking a temperature range as large as possible. The NaN1 system was also studied in THF. A few remarks should be made on some of the systems.

Precipitation occurs in the systems Li-N1-DME, Na-N1-THF, Na-N1-DME and $\mathrm{K}-\mathrm{Nl}-\mathrm{DME}$ at temperatures lower than $0^{\circ},-20^{\circ}, 0^{\circ}$ and $-40^{\circ} \mathrm{C}$, respectively. Below these temperatures no linewidth and contact shifts measurements were performed, except for Li-N1-DME, where the contact shifts were also measured below the precipitation temperature.

The experimentally determined values of the alkali N.M.R. h.f.s.c. are plotted as a function of temperature in figures 5 and 6 . The E.S.R. data in figure 5 are taken from the work of Dodson and Reddoch [4] and Hirota [5]. 

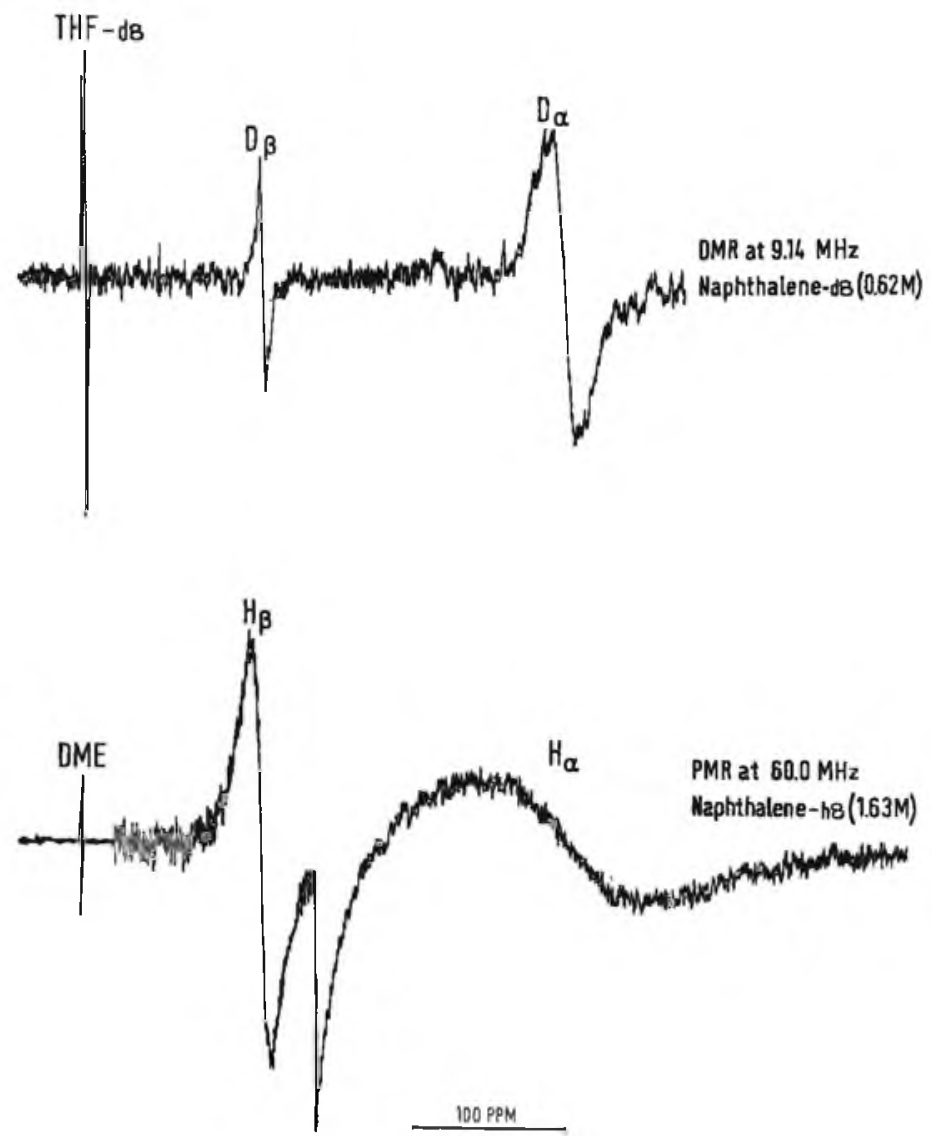

Figure 4. ${ }^{1} \mathrm{H}$ and $2 \mathrm{D}$ N.M.R. spectrum of a solution containing $1 \cdot 63 \mathrm{M} \mathrm{Nl}-\mathrm{h} 8$ and $0.62 \mathrm{M}$ Nl-d8 in DME, which was completely reduced with Na. As internal reference about 5 volume per cent $\mathrm{THF}-\mathrm{d} 8$ was added. The peculiar shape of the reference signal THF-d8 was caused by modulation effects.

The linewidths are plotted as a function of $\eta_{0} / T$ in figures 7 and 8 and were all obtained on completely reduced solutions. For the present we have used the viscosity of the pure solvent. According to equation (14) this is not completely correct. However, if the term between brackets varies only slightly with the temperature (evidence exists to justify this assumption [25-27]) the use of $\eta_{0}$ instead of $\eta$ will affect the slope of the plots by a constant factor only.

\section{Discussion}

\subsection{Hyperfine splitting constants}

\subsubsection{Proton hyperfine splitting constants}

Judging from the data in table 1, the proton N.M.R. h.f.s.c. agree with the results of E.S.R. measurements [28]. The small differences between the N.M.R. and the E.S.R. h.f.s.c. of a particular proton are not significant and may be expected in view of the different conditions [4] under which the measurements are performed, i.e. the E.S.R. experiments in dilute solutions, $\left(\simeq 10^{-4} M\right)$, and the N.M.R. in 


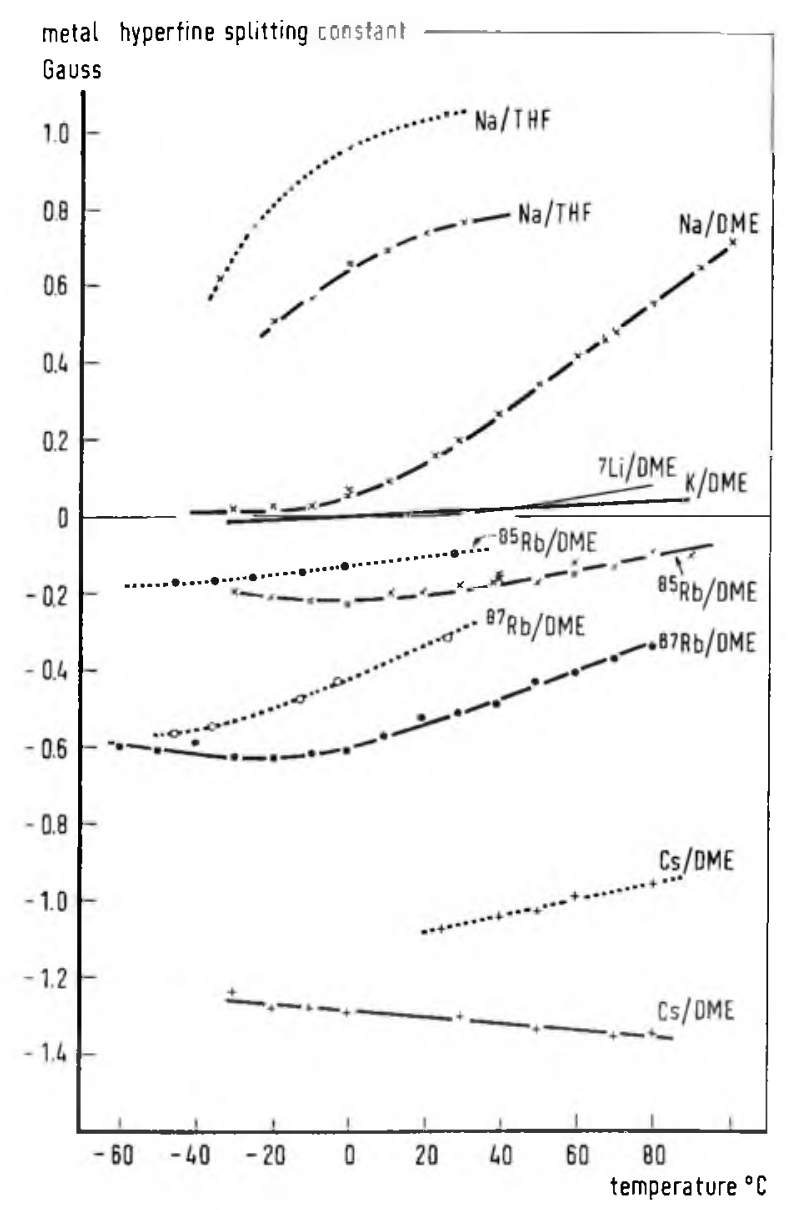

Figure 5. Temperature dependence of the alkali metal h.f.s.c. observed for the alkali naphthalene ion pairs in THF and DME. Solid lines (-) refer to N.M.R. data, dashed lines (...........) to E.S.R. results of Hirota [5] and Dodson and Reddoch [4].

concentrated solutions $(\simeq 1 \mathrm{M})$. The negative signs of the h.f.s.c. are accounted for by the spin polarization mechanism.

\subsubsection{Alkali hyperfine splitting constants}

Figure 5 shows that the sign of the $\mathrm{Li}$ and $\mathrm{Na}$ h.f.s.c. is positive, that of the $\mathrm{Rb}$ and $\mathrm{Cs}$ negative, whereas the h.f.s.c. for $\mathrm{K}$ is either negative or positive, depending on the temperature. The results demonstrate that one can use with rather good confidence the arguments of Dodson and Reddoch [4] and of Hirota [5] for predicting the sign.

The ratio of the ${ }^{6} \mathrm{Li}$ and $7 \mathrm{Li}$ h.f.s.c. is equal to the ratio of the gyromagnetic moments $\gamma{ }^{6} \mathrm{Li} / \gamma{ }^{7} \mathrm{Li}$ within experimental error (figure 6).

The plot of the $\mathrm{Na}$ h.f.s.c. versus the temperature (figure 5) for the system $\mathrm{Na}-\mathrm{N} 1-\mathrm{DME}$ is strongly curved. It suggests that solvent separated ion pairs exist at low temperatures, changing to contact ion pairs at high temperatures. This view is supported by the data obtained in THF. In this solvent, which is less solvating 


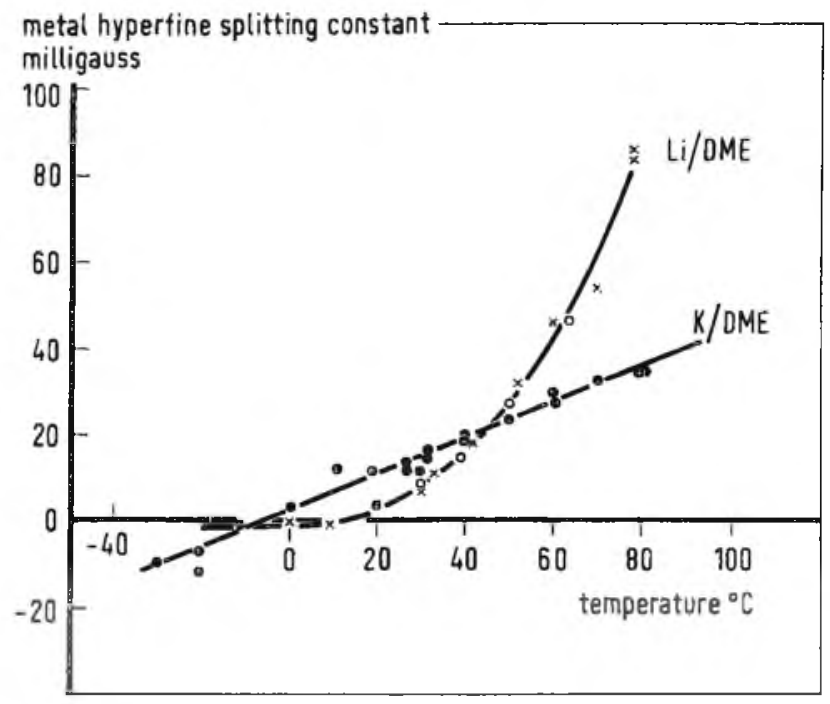

Figure 6. Li and K N.M.R. h.f.s.c., measured on solutions of $\mathrm{Li}$ and $\mathrm{KN} 1$ in DME, versus the temperature $x=a{ }^{7} \mathrm{Li}, O=a^{8} \mathrm{Li} \cdot\left(\gamma^{7} \mathrm{Li} / \gamma^{8} \mathrm{Li}\right)$.

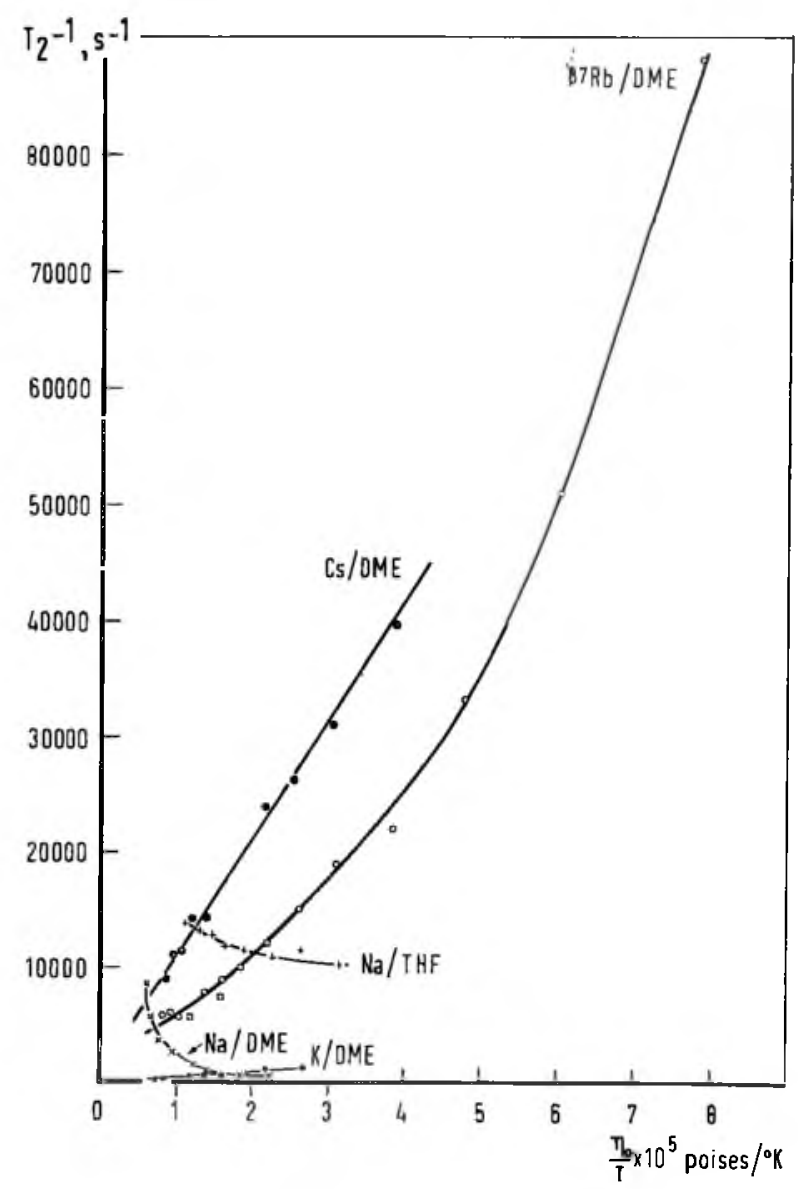

Figure 7. Alkali metal N.M.R. linewidths $\left(T_{2}{ }^{-1}\right)$ versus $\eta_{0} / T$. 


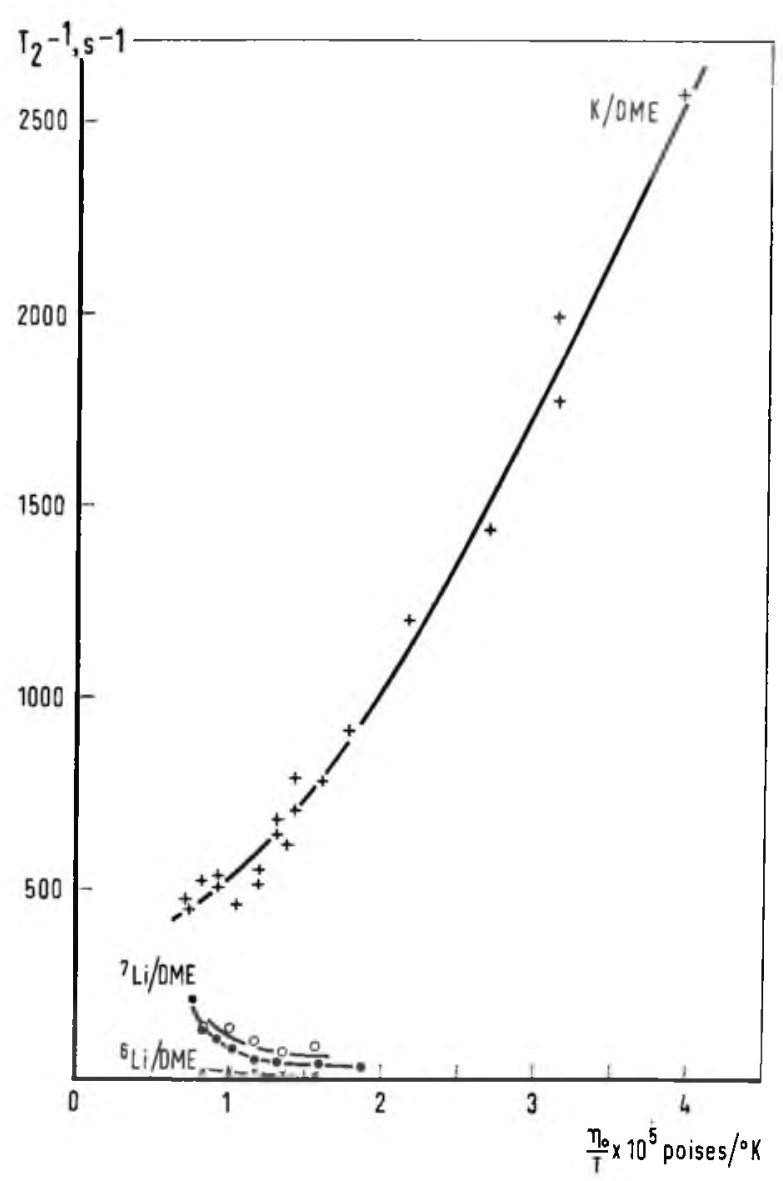

Figure 8. Alkali metal N.M.R. linewidths $\left(T_{2}^{-1}\right)$ versus $\eta_{0} / T . \quad x=\left(T_{2}-1\right){ }^{6} \mathrm{Li}$, $\mathrm{O}=\left(T_{2}{ }^{-1}\right)^{7} \mathrm{Li} \cdot\left(\gamma^{7} \mathrm{Li} / \gamma^{6} \mathrm{Li}\right)^{2}$.

than DME, the Na h.f.s.c. varies only slightly with temperature and approaches a limiting value. This behaviour can be explained by the existence of contact or tight ion pairs over the entire investigated temperature range.

The sign reversal observed for the $\mathrm{K}$ h.f.s.c. can best be rationalized by assuming the existence of contact ion pairs over the entire temperature range studied. For solvent separated ion pairs one should not expect a sign reversal of the spin density at the alkali nucleus.

From the temperature dependence of the $\mathrm{Rb}$ h.f.s.c. one may conclude that $\mathrm{RbNl}$ forms contact ion pairs at high temperatures (above about $-20^{\circ} \mathrm{C}$ ) and solvent separated ion pairs may start to form at low temperatures.

The almost constant value of the Cs h.f.s.c. is typical for contact ion pairs, of which the structural configuration does not alter much with the temperature.

Two general features emerge from the data. Firstly the tendency to form contact ion pairs is enhanced with increasing radius of the alkali ion, due to decreasing solvation. Secondly the h.f.s.c. tend to become negative for the larger cations. This has also been observed for the alkali biphenyl ion pairs $[8,12]$.

In a forthcoming article we will present an explanation for this. 


\subsection{1. ${ }^{1} \mathrm{H}$ and ${ }^{2} \mathrm{D}$ linewidth}

\subsection{Linewidths}

It can be shown $[12,15]$ that for large h.f.s.c., as encountered in the naphthalene negative ion, the only important contribution to the linewidth is the Fermi contact interaction; the linewidth should then be proportional to $a^{2}$. Therefore we have included in table 1 between brackets the ratio of the linewidths and the ratio of the squares of the N.M.R. h.f.s.c., all normalized to the values referring to the $\beta$-proton peak. Within the accuracy of the measurements the ${ }^{1} \mathrm{H}$ linewidths, indeed, are found to be proportional to $a^{2}$. Using equation (9) and the value of $T_{2}{ }^{-1}$ reported in table 1 we calculated for $\tau_{\mathrm{e}}$ a value of $(1 \cdot 8 \pm 0 \cdot 1) \times 10^{-11} \mathrm{~s}$.

Figure 3 shows that the linewidth is linearly related to the inverse of the concentration of negative ions. As pointed out in $\S 3.2$, this is to be expected in cases where the Fermi contact interaction predominates over other type of interactions, so that the linewidth is determined by $\left(T_{2}{ }^{-1}\right)_{\text {F.C. }}$. For an analysis of the $\mathrm{Na}$ linewidths we shall make use of figure 3 , in order to determine $\tau_{\mathrm{e}}$.

According to equations (6), (7) the contribution of the magnetic interactions to $T_{2}{ }^{-1}$ of the ${ }^{2 \mathrm{D}}$ resonance peaks will become $\left(\gamma_{\mathrm{H}} / \gamma_{\mathrm{D}}\right)^{2} \approx 40$ times smaller than those to $T_{2}{ }^{-1}$ of the ${ }^{1} \mathrm{H}$ peaks, whereas equations (1), (2) predict the contact shift to remain the same, if the experiments are performed at the same magnetic field as the ${ }^{1} \mathrm{H}$ experiments. This will result in a better resolution of the ${ }^{2} \mathrm{D}$ spectrum, as is clearly manifested in figure 4 .

From table 1 it is clear that the ${ }^{2} \mathrm{D}$ linewidth is not proportional to $a^{2}$. We ascribe this deviation to a quadrupole contribution to the linewidth. Since the experiments were carried out on the same (mixed) sample, a reliable value for the quadrupole contribution to the linewidth can be obtained by subtracting the ${ }^{1} \mathrm{H}$ linewidth from the ${ }^{2} \mathrm{D}$ linewidth, accounting for the difference in nuclear magnetic moments. This yields a quadrupole contribution to the linewidth of the $\alpha$ and $\beta$ deuterons:

$$
\left(T_{2}^{-1}\right)_{Q, \alpha}=69 \pm 30 \mathrm{~s}^{-1} \text { and }\left(T_{2}^{-1}\right)_{Q, \beta}=46 \pm 7 \mathrm{~s}^{-1} \text {, }
$$

respectively. For a $\mathrm{C}-\mathrm{D}$ bond [29] the quadrupole coupling constant $\left(e^{2} q Q / h\right)$ is about 0.18 MHz. The linewidths of the $\alpha$ and $\beta^{2} \mathrm{D}$ peaks in the N.M.R. spectrum of neutral N1-d8 in DME at $+30^{\circ} \mathrm{C}$ amount to $7 \cdot 8 \pm 0.6 \mathrm{~s}^{-1}$. Using equation (11) and the above-mentioned value for the quadrupole coupling constant one derives $\tau_{\mathrm{I}}=(1.6 \pm 0.2) \times 10^{-11} \mathrm{~s}$, which is in fair agreement with $\tau_{\mathrm{r}}=1.9 \times 10^{-11} \mathrm{~s}$, derived from the Debye-Einstein relation [22]. Substituting the former value of $\tau_{\mathrm{r}}$ in equation (11) and using the quadrupole contribution (15) to the $\alpha$ and $\beta^{2} \mathrm{D}$ linewidth, one finds

$$
\left(\frac{e^{2} q Q}{h}\right)_{\alpha}=0.54 \pm 0.16 \mathrm{MHz}, \quad\left(\frac{e^{2} q Q}{h}\right)_{\beta}=0.44 \pm 0.07 \mathrm{MHz} .
$$

If the value for $\tau_{\mathrm{r}}$ is not largely in error [30], then the above-calculated quadrupole coupling constants exceed the values reported in the literature for the $\mathrm{C}-\mathrm{D}$ bond by about a factor of three. For the time being it is not fully clear whether this additional contribution is from inter or intramolecular origin. If it originates from the counter ions and/or solvent molecules the field gradient should depend on the structure of the ion pair, and hence on the nature of the solvent. This will be the subject of future investigations.

Also, here, a linear relationship has been observed between the linewidth and the inverse of the concentration (figure 3 ) as one might expect from the predominant 
Fermi contact contribution to the linewidth. In a recent note [31] we suggested that experiments carried out on mixed samples containing deuterated and non-deuterated radicals might reveal a spin relaxing effect [32], i.e. the linewidths of the ${ }^{1} \mathrm{H}$ and ${ }^{2} \mathrm{D}$ resonance peaks are narrowed by the spin-spin interaction between the deuterated and the non-deuterated radical. The expectations are borne out by the present experiments. Comparing the linewidths from the mixed sample (see table I) with those measured for individual samples Nl-h8 and Nl-d8 (also at 2.25 M cf. figure 3), one notices that they are the same. Without a spin relaxing effect, the ${ }^{1} \mathrm{H}$ and ${ }^{2} \mathrm{D}$ linewidths of the mixed sample should be much larger than observed.

\subsubsection{Alkali linewidth}

A complete analysis of the alkali linewidths is hampered by the fact that the structure of the ion pair is unknown. For this reason exact calculations on the anisotropic dipolar and quadrupolar interaction are impossible. However, an upper limit of the linewidth due to the Fermi contact interaction can always be given, making use of the measured h.f.s.c. and the correlation time $\tau_{\mathrm{e}}$, obtained from the proton experiments. If resonances of two isotopes can be studied (as was done for $\mathrm{Li}$ and $\mathrm{Rb}$ ) a distinction [8] can be made between the magnetic and the quadrupole interactions.

$L i-N L-D M E$. The ${ }^{7} \mathrm{Li}$ linewidth is not proportional to $\eta_{0} / T$ [33], but increases rapidly at higher temperature (figure 8). At $+40^{\circ} \mathrm{C}$ the linewidth and the ${ }^{7} \mathrm{Li}$ h.f.s.c. are $T_{2}^{-1}=39 \mathrm{~s}^{-1}$ and $a=18$ milligauss, respectively. From figure 3 and equation (13) it follows that for a $1 \mathrm{M}$ concentration of NaNl, $\tau_{\mathrm{e}}=6 \times 10^{-11} \mathrm{~s}$ at $+40^{\circ} \mathrm{C}$. Taking the same value for $\tau_{\mathrm{e}}$ for a $1 \mathrm{M}$ solution of LiNl we calculate, using equation (9), $\left(T_{2}{ }^{-1}\right)_{\text {F.C. }}=1.5 \mathrm{~s}^{-1}$. Hence we conclude that the Fermi contact interaction does not suffice to explain the total linewidth. The experiments with the isotope ${ }^{6} \mathrm{Li}$ enable us to analyse the linewidths further $[8,12]$. In figure 8 some points are given, referring to the ${ }^{6} \mathrm{Li}$ linewidth. These points are obtained by multiplying the values for $T_{2}{ }^{-1}$ by the square of the ratio of the gyromagnetic momentst, thus normalizing the linewidth of ${ }^{6} \mathrm{Li}$ to that of ${ }^{7} \mathrm{Li}$.

If quadrupole contributions to $T_{2}^{-1}$ are negligible, the points should lie on the solid line, representing $T_{2}{ }^{-1}$ for ${ }^{7} \mathrm{Li}$. It appears that the points lie above the solid line, but this is probably not significant since it should be realized that corrections for magnetic field inhomogeneity and intermolecular dipolar relaxation to the narrow ${ }^{6} \mathrm{Li}$ lines are of considerable importance. Keeping this in mind, one may conclude that the quadrupole contributions to $T_{2}{ }^{-1}$ are very small (otherwise the points should fall below the solid line, since the quadrupole moment for ${ }^{7} \mathrm{Li}$ is much larger than for ${ }^{6} \mathrm{Li}[34]$ ), so that the most important relaxation mechanism for the $\mathrm{Li}$ linewidths is the anisotropic intramolecular dipolar interaction. This interaction is expected to increase with temperature as the distance between $\mathrm{Nl}^{-}$and $\mathrm{Li}^{+}$due to decreased solvent interaction becomes smaller. This explanation is consistent with the increase in the Li h.f.s.c. as the temperature is raised (figure 6).

$N a-N l-D M E, T H F$. In table 2 the linewidth data are listed for two temperatures, $+30^{\circ} \mathrm{C}$ and $+90^{\circ} \mathrm{C} . \quad\left(T_{2}{ }^{-1}\right)_{\text {F.C. }}$ was calculated from equation (9) and the tabulated values for $a$ and $\tau_{\mathrm{e}}$. The values for $\tau_{\mathrm{e}}$ were calculated with the help of figure 3, equation (9) and equation (13). One notices that the main part of the

† The gyromagnetic ratios were taken from Varian N.M.R. table, 1965, fifth edition. 


\begin{tabular}{|c|c|c|c|c|c|c|}
\hline & $\begin{array}{c}T \\
\left({ }^{\circ} \mathrm{C}\right)\end{array}$ & $\begin{array}{c}a_{\mathrm{Na}} \\
(\mathrm{gauss})\end{array}$ & $\begin{array}{c}\tau_{\mathrm{e}} \\
(\mathrm{s})\end{array}$ & $\begin{array}{c}T_{2^{-1}} \\
\left(\mathrm{~s}^{-1}\right)\end{array}$ & $\begin{array}{c}\left(T_{2}{ }^{-1}\right)_{\mathbf{F . C .}} \\
\left(\mathrm{s}^{-1}\right)\end{array}$ & $\begin{array}{c}\left(T_{2^{-1}}\right)_{\mathbf{D}}+\left(T_{2}{ }^{-1}\right)_{\mathbf{Q}} \\
\left(\mathrm{s}^{-1}\right)\end{array}$ \\
\hline \multirow{2}{*}{$\mathrm{DME}$} & +30 & $0 \cdot 200$ & $7 \times 10^{-11}$ & 1200 & 200 & 1000 \\
\hline+90 & $0 \cdot 634$ & $3 \times 10^{-11}$ & 5900 & 900 & 5000 \\
\hline THF & +30 & $0 \cdot 764$ & - & 12900 & - & - \\
\hline
\end{tabular}

Table 2. Analysis of the sodium linewidth.

linewidth arises from dipolar and quadrupolar interactions. The large increase of the linewidth when DME is replaced by THF is accompanied by a large increase of the h.f.s.c., indicating contact ion pair formation in THF. The $\mathrm{Na}$ ion is then closer to the aromatic plane, which enhances the magnetic contributions to the linewidth. The increase of the h.f.s.c. with the temperature (figure 5) runs parallel with the increase of the linewidth (figure 7).

$K-N l-D M E$. The small value of the h.f.s.c. renders it unlikely that magnetic interactions will contribute appreciably to the linewidth. In fact, an unrealistic value for $\tau_{\mathrm{e}}$ of $4 \times 10^{-8} \mathrm{~s}$ is needed to account for the measured $T_{2}^{-1}$ by the Fermi contact interaction. Furthermore, the $T_{2}{ }^{-1}$ versus $\eta_{0} / T$ curve in figure 8 does not show a singularity at the point, where the h.f.s.c. is equal to zero. Ascribing the total linewidth to quadrupole interactions [12] and using for $\tau_{\mathbf{r}}$ an upper and lower limit of $10^{-10}$ and $10^{-11} \mathrm{~s}$ respectively, one finds that $e^{2} q Q / h$ lies in the range 1.3-4.1 MHz, which is quite acceptable if one compares it with data obtained on other ionic $\mathrm{K}$ compounds [35].

$R b-N l-D M E$. Figure 7 illustrates that the temperature dependence of the ${ }^{87} \mathrm{Rb}$ linewidth is mainly determined by the change in $\eta_{0} / T$, which indicates that the structure of the ion pair does not change dramatically in the temperature range studied. The linewidth plot of ${ }^{85} \mathrm{Rb}$ shows a similar behaviour (not shown on figure 7) and almost coincides with the line for ${ }^{87} \mathrm{Rb}$. This fact points to the presence of quadrupole contributions to the linewidth, in contrast to what has been found for the linewidths of the two $\mathrm{Li}$ isotopes.

A complete analysis of the $\mathrm{Rb}$ linewidth has been performed at $+30^{\circ} \mathrm{C}$. This can be accomplished by writing equation (5) for the two isotopes ${ }^{87} \mathrm{Rb}(\mathrm{A})$ and ${ }^{85} \mathrm{Rb}$ (B) as [8]:

$$
\begin{gathered}
\left(T_{2}^{-1}\right)_{\mathrm{A}}=c_{1} \gamma^{2}{ }_{\mathrm{A}}+c_{2} f\left(I_{\mathrm{A}}\right)\left(e Q_{\mathrm{A}}\right)^{2}, \\
\left(T_{2}{ }^{-1}\right)_{\mathrm{B}}=c_{1} \gamma_{\mathrm{B}}{ }^{2}+c_{2} f\left(I_{\mathrm{B}}\right)\left(e Q_{\mathrm{B}}\right)^{2} .
\end{gathered}
$$

The first term on the right-hand side of these equations represents

$$
\left(T_{2}^{-1}\right)_{\text {F.C. }}+\left(T_{2}^{-1}\right)_{\mathrm{D}}
$$

while the second one represents $\left(T_{2}{ }^{-1}\right)_{\mathrm{Q}}$. Values for $f\left(I_{\mathrm{A}}\right)$ and $f\left(I_{\mathrm{B}}\right)$ can be obtained from equation (8) by substituting the nuclear quantum numbers $I_{\mathrm{A}}$ and $I_{\mathrm{B}}$ of the respective isotopes. To solve the equations for $c_{1}$ and $c_{2}$, it is sufficient to know the ratios of $\gamma_{\mathrm{A}} / \gamma_{\mathrm{B}} \dagger$ and $Q_{\mathrm{A}} / Q_{\mathrm{B}}[36]$ and to measure $\left(T_{2}{ }^{-1}\right)_{\mathrm{A}}$ and $\left(T_{2}{ }^{-1}\right)_{\mathrm{B}}$. The result of the analysis is given in table 3 .

+ The gyromagnetic ratios were taken from Varian N.M.R. table, 1965, fifth edition. 


\begin{tabular}{|c|c|c|c|c|}
\hline Isotope & $\left(T_{\left.2^{-1}\right)\left(\mathrm{s}^{-1}\right)}\right.$ & $\left(T_{\left.2^{-1}\right)_{Q}\left(\mathrm{~s}^{-1}\right)}\right.$ & $\left(T_{2^{-1}}\right)_{\mathbf{D}_{+} \text {F.C. }}\left(\mathrm{s}^{-1}\right)$ & $\left(T_{2^{-1}}\right)_{\text {F. C. }}\left(\mathrm{s}^{-1}\right)$ \\
\cline { 2 - 5 }${ }^{85} \mathrm{Rb}$ & $7780 \pm 150$ & $7670 \pm 190$ & $106 \pm 35$ & $112 \pm 28$ \\
${ }^{87} \mathrm{Rb}$ & $8700 \pm 330$ & $7480 \pm 180$ & $1210 \pm 400$ & $1010 \pm 260$ \\
\hline
\end{tabular}

Table 3. Analysis of the rubidium linewidth at $+30^{\circ} \mathrm{C}$.

In order to establish a further distinction between the magnetic interactions, we have measured from the same sample the $\beta$-proton resonance peak. Using equation (9) an upper limit for $\tau_{\mathrm{e}}$ was found, $\tau_{\mathrm{e}} \leqslant(4 \cdot 7 \pm 0 \cdot 7) \times 10^{-11} \mathrm{~s}$. Subsequently $\left(T_{2}{ }^{-1}\right)$ F.c. was calculated with the help of equation (9), substituting in this formula the $\mathrm{Rb}$ h.f.s.c. The table demonstrates that the anisotropic dipolar interaction is quite unimportant and that the quadrupole relaxation governs the ${ }^{85} \mathrm{Rb}$ linewidth for almost 100 per cent and the linewidth of ${ }^{87} \mathrm{Rb}$ for about 85 per cent.

$C s-N l-D M E$. Figure 7 shows that the Cs linewidth varies linearly with $\eta_{0} / T$ in agreement with the insensitivity of the Cs h.f.s.c. with a change of temperature (see figure 5). At $T=+30^{\circ} \mathrm{C}$ the linewidth and the h.f.s.c. of $\mathrm{Cs}$ amount to $T_{2}{ }^{-1}=13000 \mathrm{~s}^{-1}$ and $a=-1.31$ gauss respectively. A quadrupole contribution to the Cs linewidth can be safely neglected, on account of the very small quadrupole moment [37].

Extrapolating the results of the $\mathrm{Rb}$ linewidth analysis to $\mathrm{Cs}$, we expect, that the Fermi contact interactions will determine the linewidth. This was found also by Canters et al. [9] for the Cs-biphenyl ion pairs. If we ascribe the total linewidth to this interaction, we calculate an upper limit for $\tau_{\theta}$ of $1.0 \times 10^{-10} \mathrm{~s}$.

The near constancy of the h.f.s.c. with temperature and the proportionality of $T_{2}{ }^{-1}$ with $\eta_{0} / T$ can be taken as characteristic for tight or contact ion pairs.

After completing this manuscript a short paper appeared by Takeshita and Hirota [38] on sign determination of alkali h.f.s.c. in some alkali radical ion pairs by, N.M.R. Their results for $\mathrm{Na}$ and $\mathrm{Cs}$-naphthalenide measured at one temperature agree qualitatively with ours.

The authors wish to thank Professor Dr. J. Smid for the critical reading of this manuscript. The present investigations have been carried out under the auspices of the Netherlands Foundation of Chemical Research (S.O.N.) and with the aid of the Netherlands Organization for the Advancement of Pure Research (Z.W.O.).

\section{REFERENCES}

[1] Atherton, N. M., and Weissman, S. I., 1961, F. Am. chem. Soc., 83, 1330.

[2] Symons, M. C. R., 1967, f. phys. Chem., 71, 172.

[3] De Boer, E., 1965, Recl Trav. chim. Pays-Bas, 84, 609.

[4] Dodson, C. L., and Rednoch, A. H., 1968, F. chem. Phys., 48, 3226.

[5] Hirota, N., 1968, F. Am. chem. Soc., 90, 3603.

[6] Herold, B. J., Correia, A. F. N., and Dos Santos Veiga, J., 1965, Ұ. Am. chem. Soc., 87, 2661.

[7] Warhurst, E., and WiLde, A. M., 1969, Trans. Faraday Soc., 65, 1413.

[8] Canters, G. W., De Boer, E., Hendriks, B. M. P., and van Willigen, H., 1968, Chem. Phys. Lett., 1, 627.

[9] Canters, G. W., De Boer, E., Hendriks, B. M. P., and Klaassen, A. A. K., 1969, Proc. Coll. Ampère $X V$, edited by $\mathrm{P}$. Averbuch (North-Holland. Publishing Co.), p. 242. 
[10] Reuben, J., and Fiat, D., 1969, F. Am. chem. Soc., 91, 1242.

[11] Paul, D. E., Lipkin, D., and Weissman, S. I., 1956, Y. Am. chem. Soc., 78, 116.

[12] Canters, G. W., 1969, Thesis, University of Nijmegen, Nijmegen, The Netherlands.

[13] MeYkrs, O. E., and Putzer, E. J., 1959, Y. appl. Phys., 30, 1987.

[14] Haworth, O., and Richards, R. E., 1966, Progress in Nuclear Magnetic Resonance Spectroscopy (Pergamon Press), Chap. 1.

[15] Canters, G. W., and de Boer, E., 1967, Molec. Phys., 13, 395.

[16] De Boer, E., and Maclean, C., 1965, Molec. Phys., 9, 191; 1966, f. chem. Phys., 44, 1334.

[17] Carrington, A., and McLachlan, A. D., 1967, Introduction to Magnetic Resonance (Harper \& Row), Chap. XIII.

[18] De Bokr, E., Grotens, A. M., and Smm, J., 1970, Y. Am. chem. Soc., 92, 4742.

[19] Blozmbergen, N., 1957, J. chem. Phys., 27, 572.

[20] Solomon, I., 1955, Phys. Rev., 99, 559.

[21] Abragam, A., 1961, The principles of Nuclear Magnetism (Clarendon Press), Chap. 8.

[22] Carrington, A., and Maclachlan, A. D., 1967, Introduction to Magnetic Resonance (Harper \& Row), p. 189.

[23] PAke, G. E., and Tuttle, T. R. Jr., 1959, Phys. Rev. Lett., 3, 423.

[24] Jones, G., and Dole, M., 1929, Э. Am. chem. Soc., 51, 2950.

[25] KaY, R. L., Viruccio, T., ZawoYski, L., and Evans, D. F., 1966, J. phys. Chem., 70, 2336.

[26] Millero, F. J., 1968, 7. phys. Chem., 72, 3209.

[27] Canters, G. W., 1969, Thesis, University of Nijmegen, Nijmegen, The Netherlands, p. 57.

[28] Segal, B. G., Reymond, A., and Fraenkri, G. K., 1969, f. chem. Phys., 51, 1336.

[29] Ellis, D. M., and BJorkstaM, J. L., 1967, Ұ. chem. Phys., 46, 4460.

[30] Canters, G. W., 1969, Thesis, University of Nijmegen, Nijmegen, The Netherlands, p. 98.

[31] Canters, G. W., Hendriks, B. M. P., and De Bokr, E., 1970, F. chem. Phys., 53, 445.

[32] Kreilick, R. W., 1968, J. Am. chem. Soc., 90, 2711.

[33] Carvajal, C., Tölle, K. J., Smid, J., and Szwarc, M., 1965, J. Am. chem. Soc., 87, 5548. $\eta_{0}$ is taken from this work.

[34] Wharton, L., Gold, L. P., and Klemperer, W., 1964, Phys. Rev. B, 133, 270. $Q^{\circ} \mathrm{L} 1 / Q^{7} \mathrm{~L}=0.0176$.

[35] DaS, T. P., and Hahn, E. L., 1958, Solid St. Physics ,Suppl. I, Nuclear Quadrupole Resonance Spectroscopy, (Academic Press Inc.), p. 158.

[36] MgYkR-Berkhout, U., 1965, Z. Phys., 141, 185. $Q^{85}{ }_{\mathrm{Rb}} / Q^{87} \mathrm{Rb}=2 \cdot 00669$.

[37] Buck, P., Rabi, I. I., Senitzky, B., 1956, Phys. Rev., 104, 553.

[38] Takrshita, T., and Hirota, N., 1969, Chem. Phys. Lett., 4, 369. 